\title{
Transient interactions between biomolecules
}

\author{
Irene Díaz-Moreno • Miguel A. De la Rosa
}

Received: 13 June 2011/ Accepted: 22 June 2011/Published online: 13 July 2011

(C) European Biophysical Societies' Association 2011

The ultimate goal of cellular biology and biochemistry is to penetrate into and comprehend the complexity of the molecular interaction networks existing in the living cell so as to provide a molecular rationale for understanding the details of cell (mis)functioning and diseases. Indeed, specific protein-protein and protein-nucleic acid interactions underlie virtually every process in the cell, regulating cell metabolism and determining cell life and death.

Such interactions between biomolecules occur on a wide range of timescales. Stable complexes, with lifetime ranging from minutes to days, involve high-affinity and high-specificity binding. Amongst many others, examples are irreversible enzyme inhibition by inhibitors and assembly of proteins supporting the cell ultrastructure. Weak complexes, with lifetime in the second-microsecond range, are formed when fast turnover or reversible multicomponent assembly is required.

The topic of this Special Issue of European Biophysics Journal, entitled "Transient Interactions between Biomolecules", is focussed on short-lived complexes, in which a fine balance between specificity of binding and high turnover rate is sought. Actually, most of these complexes are characterized by equilibrium dissociation constants within the $\mu \mathrm{M}$ or even $\mathrm{mM}$ range. An important question arises regarding how such weak interactions guarantee specificity between partners high enough to be relevant from the biological point of view. Different systems may rely on

Special Issue: Transient interactions in biology.

I. Díaz-Moreno $(\square) \cdot$ M. A. De la Rosa

Instituto de Bioquímica Vegetal y Fotosíntesis, cicCartuja,

Universidad de Sevilla-CSIC, Americo Vespucio 49,

41092 Sevilla, Spain

e-mail: idiazmoreno@us.es different solutions. In some cases, multiple interactions may be involved, while in others a kinetic trap is possible, as in the recognition between the ribosome and transfer RNA (tRNA). Whatever the solution is, the transient interaction has to reach a compromise between specificity and fast dissociation.

Intriguingly, contrary to what one might think, these molecular recognition mechanisms are not uncommon and play a key role in many biological processes, namely the electron transfer chains in respiration and photosynthesis and the cell signalling cascades involving kinases and phosphatases. Our better molecular understanding of the different cellular processes has led to the growing realization that transient and reversible interactions are necessary for a very broad range of processes, such as assembling post-transcriptional regulatory complexes and regulating messenger RNA (mRNA) metabolism from splicing to nuclear export.

Communication between biomolecules through specific binding interfaces is essential to transfer information in an efficient way. However, such interfaces represent a small fraction of the total surface area of the interacting partners, and so their encounter and further proper interaction become a non-trivial task. To reduce the conformational search space on the path to the specific complex, it has recently proposed that complex formation, especially that involving transient interactions, proceeds via a two-step mechanism. The first step yields the so-called encounter complex, which is the result of the weak association between partners via diffusion-controlled intermolecular collisions. During the short-lived encounter phase, the two partners remain largely solvated and adopt an ensemble of different orientations located in multiple local free energy minima of a two-dimensional energy landscape on the surface. Electrostatics dominates this state. The subsequent 
rearrangement along the energy landscape, based on translations and rotations of the two partners, lets the global free energy minimum be reached, thus resulting in a well-defined complex stabilized by a set of optimized hydrophobic and electrostatic interactions between the desolvated partners. This leads to the transition towards the so-called productive complex.

An ample series of biophysical and structural models are available to study the particular features of stable complexes, but weak complexes remain poorly understood, despite their critical role in many biological events inside the cell. Over the last few years, a number of disciplines have developed, largely independently, specific tools to analyze the transient intermolecular interactions, including nuclear magnetic resonance (NMR) paramagnetic relaxation enhancement and kinetic approaches. These tools have recently been complemented by high-throughput techniques to identify novel biomolecules that transiently bind each other. The recent advances in mass spectrometry, the development of new chemical agents and the diversity of methodological approaches make the analysis of the transient biointeractome (the so-called trans-biointeractome) more affordable. In fact, crosslinking strategies have been used to address the difficulties related to short-lived complexes.

Overall, trans-biointeractome analysis remains highly dependent on the technique employed. Therefore, the study of weakly interacting systems can be tackled in depth only by an integrative and holistic approach in research on transient macromolecular interactions combining in vitro strategies with other methods to detect such interactions within the cell.

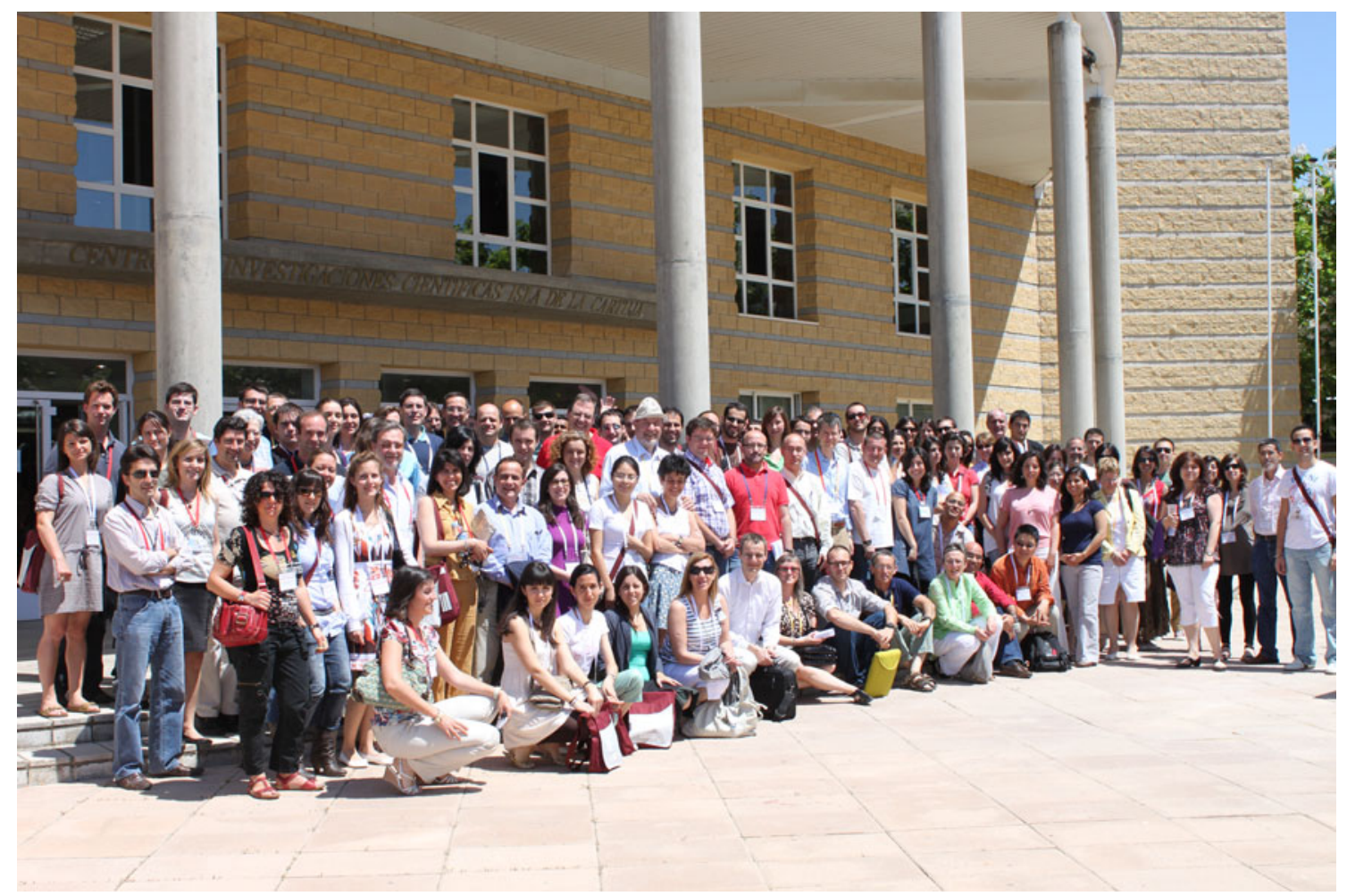

